\section{VEXAS syndrome with cutaneous nodules}

\author{
Yahya Argobi \\ Department of Medicine, King Khalid \\ University, Abha, Saudi Arabia
}

\begin{abstract}
Recently, a rare severe autoinflammatory Vacuoles, E1 enzyme, X-linked, autoinflammatory, Somatic (VEXAS) syndrome caused by somatic variants in the UBA1 gene was discovered.

The clinical features of VEXAS syndrome are heterogeneous, including highgrade fever, polychondritis and skin lesions. In 2020, Beck DB et al described an original cohort of 25 patients, of whom 22 (88\%) had cutaneous findings, namely, neutrophilic dermatitis, and vasculitis. We report a case of VEXAS syndrome and cutaneous nodules with confirmed UBA1 mutation.
\end{abstract}

\section{Introduction}

Vacuoles, E1 enzyme, X-linked, autoinflammatory, somatic (VEXAS) syndrome is a newly described, acquired autoinflammatory syndrome that can be severe and often fatal. ${ }^{1}$ Beck et al. identified 25 men with somatic mutations affecting methionine-41 (p.Met41) in UBA1, the major E1 enzyme that initiates ubiquitylation. The gene UBA1 lies on the $\mathrm{X}$ chromosome. Their somatic UBA1 variants were confirmed by Sanger sequencing. These 25 patients met clinical criteria for an inflammatory syndrome (relapsing polychondritis, Sweet's syndrome, polyarteritis nodosa, or giantcell arteritis) or a hematologic disorder (myelodysplastic syndrome or multiple myeloma) or both. ${ }^{1}$ Clinical features included fever, alveolitis, polychondritis, skin lesions (including neutrophilic dermatoses and cutaneous vasculitis), and thromboembolic disease. All participants had progressive hematologic abnormalities, including cytopenias, and myeloid dyspoiesis, but none had hematological malignancy. ${ }^{1}$ High doses of glucocorticoids were the only treatment that consistently alleviated the symptoms. Of the 25 patients, $10(40 \%)$ died from disease-related progressive anemia or respiratory failure, or complications secondary to treatment. ${ }^{1}$ Here we present a complicated case of VEXAS syndrome with cutaneous nodules.

\section{Case Report}

We report a 70-year-old male who considers himself a generally very active person until mid 2018 when he started to develop fatigue, malaise, and chills. He was found to have $3.3 \mathrm{~cm}$ lung mass; however, the biopsy was negative for malignancy. He received multiple courses of antibiotics, and few months later the repeated imaging showed that the lesion had completely resolved. His symptoms resolved only with prednisone course. He had a past medical history of treated babesiosis 7 years ago, and history of gout 15 years ago that was treated with allopurinol for few months at that time. In summer 2019, he developed multiple symptoms including fever, arthralgia and joints swellings. He was seen by a rheumatologist and this was attributed to gout given his past history. He was started on allopurinol; however, his polyarticular complaints didn't improve and he continued to have fever and chills. In Nov 2019, He was admitted to the Hospital for acute hypoxemic respiratory failure that was thought to be due to pulmonary edema, there was no clear explanation for his hypoxemia. He was noted to have fever, chills, and rash, for which work-up was largely negative. Allopurinol was discontinued due to the desire to avoid unnecessary medications. He was started on a prednisone taper starting at $40 \mathrm{mg}$ daily with significant improvement in his symptoms. Given his fevers, a temporal artery biopsy was performed, and it was negative. Multiple services were consulted including general surgery, vascular surgery, dermatology, ID, and rheumatology. He improved on prednisone and discharged home on $30 \mathrm{mg}$ daily. After he finished the prednisone course, he relapsed and was readmitted in Jan 2020 with ongoing fever and skin rash. He had a non-diagnostic bone marrow with unusual myelomonocytic population with vacuoles in erythroid and myeloid precursor cells. His fevers resolved with steroids. Extensive evaluation was negative for oncologic or infectious etiology. The periodic fever syndrome testing was also negative. He then developed a new skin rash on his trunk and cutaneous nodules on his lower extremities (Figure 1). A skin. Biopsy of one of the cutaneous nodules on his legs showed septal lymphohistiocytic panniculitis consistent with erythema nodosum. He was discharged on a prednisone taper though diagnosis remained unclear overall. He was unable to taper the dose to less than $30 \mathrm{mg}$ daily. Systemic therapies were tried including methotrexate, adalimumab, anakinra, and sarliumab but none helped.
Correspondence: Yahya Argobi, Department of Medicine, King Khalid University, One Gauraiger, PO Box 621, Aseer postal code 61421, Abha, Saudia Arabia.

Tel: +966 541485083

E-mail: yahya.derm@gmail.com

Key words: VEXAS, Autoinflammation, cutaneous nodules.

Conflict of interest: The authors declare no potential conflict of interest.

Funding: None.

Availability of data and material: Data and materials are available by the authors.

Please cite this article as: Argobi Y. VEXAS syndrome with cutaneous nodules. Dermatol Rep 2022;14:9414.

Received for publication: 2 November 2021. Accepted for publication: 2 December 2021.

This work is licensed under a Creative Commons Attribution-NonCommercial 4.0 International License (CC BY-NC 4.0).

(Copyright: the Author(s), 2022

Licensee PAGEPress, Italy

Dermatology Reports 2022; 14:9414

doi:10.4081/dr.2022.9414

Publisher's note: All claims expressed in this article are solely those of the authors and do not necessarily represent those of their affiliated organizations, or those of the publisher, the editors and the reviewers. Any product that may be evaluated in this article or claim that may be made by its manufacturer is not guaranteed or endorsed by the publisher.

Finally, genomic sequencing confirmed a somatic mutation in UBA1 gene. He was subsequently diagnosed with VEXAS syndrome and is currently under consideration for alternative treatment modalities.

\section{Discussion}

VEXAS syndrome is characterized by a somatic missense mutation affecting the UBA1 gene, resulting in the activation of the innate immune system and widespread systemic autoinflammation. The majority of Beck DB et al 25 participants had recurrent fevers, pulmonary involvement, dermatologic manifestations (including neutrophilic dermatoses and cutaneous vasculitis), macrocytic anemia, hematopoietic dyspoiesis, and bone marrow vacuolization restricted to myeloid and erythroid precursor cells. ${ }^{1}$ Our case had similar symptoms 
including skin lesions, fevers, pulmonary edema, autoinflmmation and bone marrow vacuoles with positive UBA1 mutation. Also, similar to the original cohort participants, our patient had complicated course and couldn't taper steroids below $30 \mathrm{mg}$ daily.

A Dutch case series of 12 patients with VEXAS syndrome reported constitutional symptoms and multi-organ involvement, including bone marrow; 12 of 12 patients $(100 \%)$, and skin lesions; 10 of 12 patients $(83.3 \%)^{2}$ Although VEXAS is a new syndrome with common skin lesions, the cutaneous morphological and histological features are poorly described. Nonetheless, a recent multi-center retrospective study of 8 patients with confirmed VEXAS syndrome and skin involvement including neutrophilic dermatosis and livedo racemose, showed that the dermal infiltrates of those patients was derived from the pathological myeloid clone. ${ }^{3}$ Their skin biopsies showed perivascular neutrophils and myeloid cell

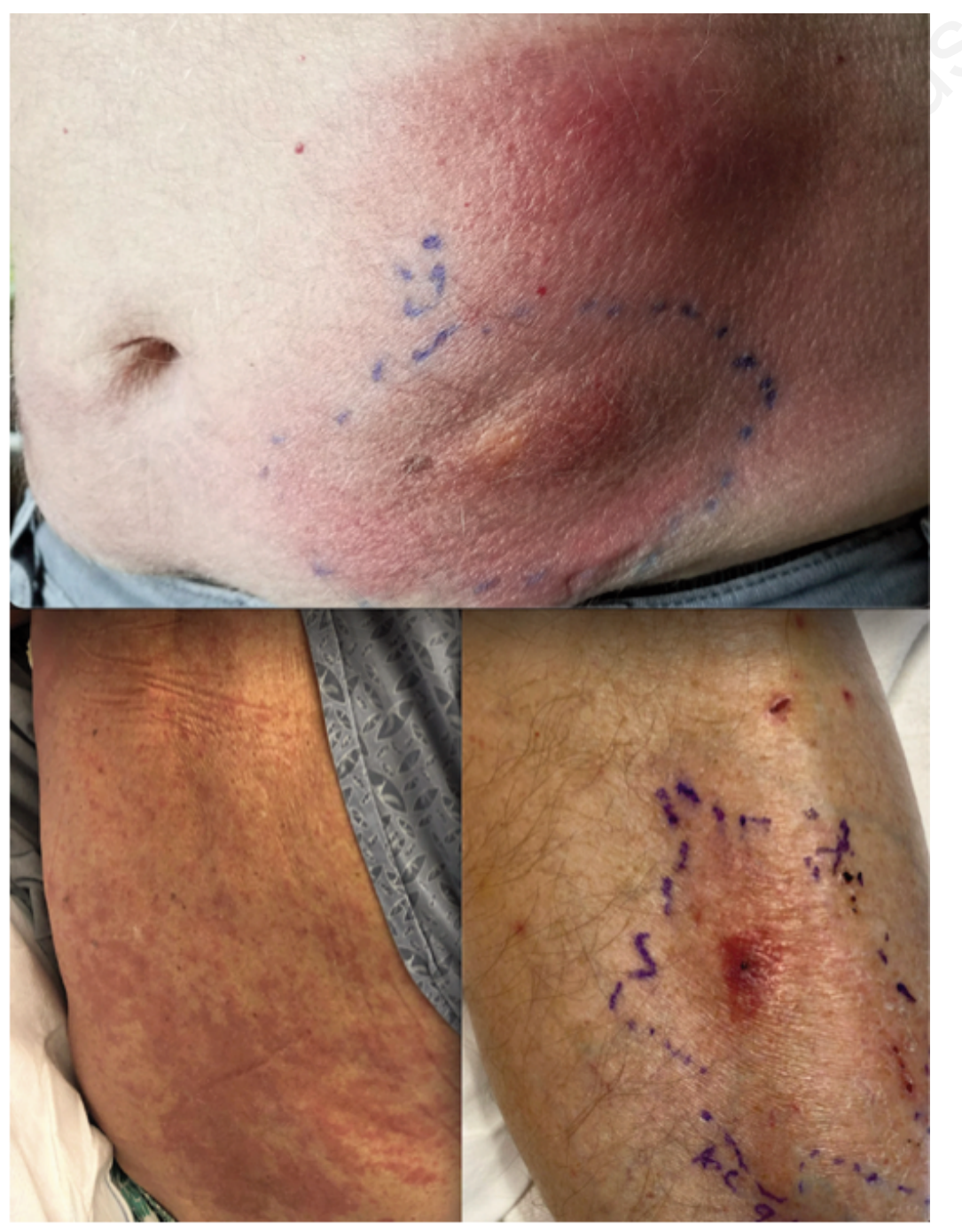

Figure 1. Edematous, erythematous plaques on the trunk and extremities. infiltration harboring UBA1 clinical variation, suggesting that the skin lesions are the result of the same pathology.

A more recent analysis of 116 French patients further characterized clinical features in VEXAS syndrome. ${ }^{[4]}$ This retrospective multi-center study was conducted in France between November 2020 and May, 2021. All cases with confirmed UBA1 somatic mutations on genetic sequencing were included. Main clinical features included skin lesions $(\mathrm{n}=97,83.6 \%)$, recurrent fever ( $\mathrm{n}=75,64.7 \%)$, and lung involvement $(n=57 ; 49.1 \%)$. Skin lesions were classified as neutrophilic dermatosis $(n=46$, $39.7 \%$ ) with pathological confirmation in only 22 cases, and cutaneous vasculitis $(\mathrm{n}=30,26 \%)$ with pathological confirmation in only 14 cases. ${ }^{4}$ Although this study showed that $83.6 \%$ had cutaneous lesions, only $47 \%$ had histological confirmation. From this study the main clinical features of VEXAS patients remain skin lesions (83.6\% vs. $88 \%$ in original description by

Beck et al. and recurrent fever $(64.7 \% v s$. $72 \%$ ). Furthermore, it expanded the previous clinical phenotype of VEXAS syndrome and reported the rare occurrence of VEXAS syndrome in women, even if the disease remains more prevalent in men. Prospective studies are required to better characterize this life-threatening syndrome and to determine the optimal management.

\section{Conclusions}

Here, we present a case of VEXAS syndrome with cutaneous nodules. Physicians should be aware of this novel disorder and consider it in adult men with features of autoinflammation, recurrent fever, hematologic abnormalities, polychondritis, and skin lesions. This disorder may follow a fatal course, with $40 \%$ of the original cohort dying from disease-related causes and or complications from long-term steroid use. Further research is needed to explore bone marrow transplantation or gene-editing therapies as potential treatment modalities.

\section{References}

1. Beck DB, Ferrada MA, Sikora KA, et al. Somatic Mutations in UBA1 and Severe Adult-Onset Autoinflammatory Disease. N Engl J Med 2020;383:262838.

2. van der Made CI, Potjewijd J, Hoogstins A, et al. Adult-onset autoinflammation caused by somatic mutations in UBA1: A Dutch case series of patients with VEXAS. J Allergy Clin Immunol 2021:S0091-6749.

3. Zakine E, Schell B, Battistella M, et al. UBA1 Variations in Neutrophilic Dermatosis Skin Lesions of Patients With VEXAS Syndrome. JAMA Dermatol 2021:e213344.

4. Georgin-Lavialle S, Terrier B, Guedon $\mathrm{AF}$, et al. Further characterization of clinical and laboratory features occurring in VEXAS syndrome in a largescale analysis of multicenter case-series of 116 French patients. Br J Dermatol. 2021. Online ahead of print. 